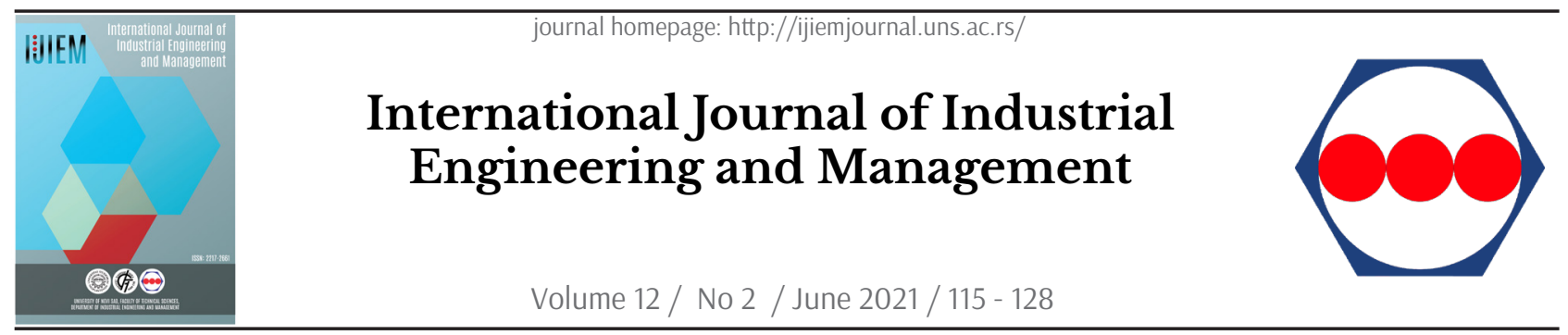

Original research article

\title{
Mass Customization Capabilities in Practice - Introducing the Mass into Customized Tech-Textiles in an SME Network
}

\author{
E. Korneeva ${ }^{a *}$, S. Hönigsberg ${ }^{b}$, F. T. Piller ${ }^{a}$ \\ a RWTH Aachen University, School of Business and Economics, Aachen, Germany; \\ ${ }^{b}$ Chemnitz University of Technology, Faculty of Economics and Business Administration, Chemnitz, Germany
}

\begin{abstract}
A B STR A C T
The German textile industry is dominated by small and medium-sized enterprises (SMEs) with limited resources and specialized skills producing customized technical textiles following an engineer-to-order approach. To expand their skills, SMEs form business networks. The development and production of customized technical textiles in networks are highly complex. The coordination requires high effort and results in inefficient and ineffective information flow, weakening the networks' competitive advantage. Following a case study approach, we accompany an SME network over three years as they develop and implement a digital col-laboration platform. We derived a framework of microfoundations of Mass Customization capabilities supporting high-order Mass Customization capabilities for customer integration, solution space development, and robust processes. Thus, we present results on how an SME network in the textile industry leverages Mass Customization capabilities to increase efficien-cy via a digital collaboration platform.
\end{abstract}

ARTICLE INFO

Article history:

Received January 11, 2021

Revised April 2, 2021

Accepted April 5, 2021

Published online May 13, 2021

Keywords:

Mass customization capabilities;

Engineer-to-order;

Digital platform;

Collaboration;

SME network;

Product configuration

*Corresponding author:

Ekaterina Korneeva

korneeva@time.rwth-aachen.de

\section{Introduction}

As a result of globalization and the removal of trade protections, the European textile industry experienced a disruption. The textile industry describes the production of textiles from the production of threads and yarns to the textile fabric. In a broader sense, the machine construction for textile production, the agricultural or chemical production of the raw materials of the textiles are also considered as preliminary stages of the textile value creation [1]. The subsequent stages of textile value creation are, for example, the clothing industry, in which clothing is designed, tailored, and traded [1]. In a narrow sense, the textile value creation is typically divided into the steps of yarn production (e.g., fiber or filament yarns), fabric production (e.g., knitted or woven fabrics), and finishing (e.g., dyeing, printing, or coating) [2]. The changes in the global market led to a decline in the textile sector in developed countries while developing countries strengthened their textile industry [3]. Forced by the competitive pressure, the European textile industry shifted towards value-adding production steps (e.g., design, fabric testing, or quality control) and knowledge-intense production, especially the development and production of technical textiles [4]. Technical textiles are designed to perform specific functions (e.g., in the automotive and 
aerospace industry) and require intensive research and development for highly customized solutions [5]. These textiles are used in a wide variety of industries and usually have to meet very stringent performance characteristics. In the automotive industry, for example, airbags and seat belts are among the technical textiles where the survival of the passengers depends on their characteristics and quality [2]. Technical textiles do not contain any parts or components, making the technical textile industry a process industry similar to the chemical and metal-processing industry, where the production knowledge is not about product modules but procedures and techniques [6]. Today, technical textile production represents over $30 \%$ of the European textile industry output [7]. Even though the textile industry in Europe experienced a sharp decline by shifting towards technical textiles, the traditional sector still holds an important position in the global market as the second-largest textile and apparel exporter after China [8]. Firms in the technical textile sector compete on the basis of constant innovation and the development of new products, as commodification has not yet taken place [5]. The technical textile industry thus pursues an engineer-to-order approach, which is characterized by a high degree of customization of the products, small production volumes and a high process complexity [9]. In order to reduce complexity, engineer-to-order firms in some cases pursue the strategy of carrying out a particularly large number of production steps in their own companies or modularizing and standardizing as many parts of the product as possible [10]. These strategies for complexity reduction in the context of engineer-to-order lead to a convergence towards a mass customization approach through higher efficiency in the production of individualized products [9]. The fragmented nature of the textile value chain, with SMEs organized in production networks, each firm performing essential production steps, makes the coordination of (technical) textile production highly complex [11]. The development and production of customized technical textiles thus require high coordination efforts along the textile value chain. Inefficient and ineffective coordination result in long development and production periods, which weaken the competitive advantage of geographical proximity and short delivery times of European textile firms against Asian competitors. The path for technical textile producers towards the efficiencies of Mass Customization is therefore particularly difficult, and the foundations of the capabilities such companies need for it are under-researched.

This paper investigates how a production net- work of four textile firms producing custom technical textiles can leverage Mass Customization (MC) principles to increase the efficiency and effectiveness in coordinating their production. The investigated firms rely on an engineer-to-order approach. In the engineer-to-order approach, the decoupling point where the customer can intervene in the design and manufacture of individual products lies upstream in the value chain [12]. By placing the customer decoupling point in the design and development stage of the value chain, the degree of customer integration is higher, and the solution space is more open [13]. Our embedded case study presents how firms can leverage MC-principles to increase the efficiency and effectiveness of an engineer-to-order approach.

The investigated firms implement a collaboration platform for textile product configuration to realize IT-supported development and production of customized technical textiles. Configurators are a common approach among firms striving towards an MC-strategy [14], [15]. However, the introduction of such technology does not automatically result in a firm's ability to execute an MC-strategy. Firms need to develop specialized capabilities to align new technologies with their goal of improved efficiency and effectiveness. Literature provides three MC-capabilities: solution space development, robust process design, and customer integration [16]. Those capabilities are high-order capabilities [17], far away from actionable suggestions for firms. Hence, the question of which concrete and actionable capabilities small and medium-sized textile enterprises with an engineer-to-order approach need to move towards MC-efficiency arises.

The literature highlights the integration of MC-capabilities of incumbent firms moving from mass production to Mass Customization [16]. However, on the other side of the continuum, we find SMEs with an engineer-to-order approach, manufacturing individual unstandardized products. Our study highlights the latter path towards the ideal state of Mass Customization [16]. We illustrate this path following the logic of the illustration of Salvador et al. [18] for firms moving from mass production to MC (cf. Figure 1).

To address our research question, we derived micro-foundations of MC-capabilities, which are closer to a firm's operational capabilities and, therefore, more actionable for firms. Micro-foundations are defined as 'the underlying individual-level and group actions that shape strategy, organization, and, more broadly, dynamic capabilities' [19]. This paper presents a framework of micro-foundations of MC-capabilities supporting the high-order MC-capabilities 
for business-to-business (B2B) firms. More specifically, we derived a subset of micro-foundations for networked engineer-to-order collaborations in the process industry from the real-world case of a textile network with four SMEs.

The remaining paper is structured as follows. First, we provide a theoretical background for Mass Customization capabilities by distinguishing between high-order capabilities and micro-foundations of Mass Customization capabilities. Then, we describe our research approach by presenting the case setting and the data collection and analysis. Third, we present our results. Therefore, we first present the Mass Customization micro-foundation framework and second, how the SME network put suited micro-foundations into practice. We close the paper with a discussion of the results and a conclusion.

\section{Background}

\subsection{Mass Customization Capabilities}

Mass Customization is defined as "producing goods and services to meet individual customer's needs with near mass production efficiency" [20]. However, as Salvador et al. [16] argue, Mass Customization is not a state where a firm knows each customer's needs precisely and fulfills those needs at mass production cost. Mass Customization is rather a process towards that ideal state, which is impossible to achieve [16]. Firms can get closer to the ideal state by incorporating three core capabilities: solution space development, robust process design, and customer integration.

A firm's solution space clearly defines what prod- uct variations it offers and what it does not. Therefore, firms need to identify product attributes among which customer needs diverge significantly [16]. Addressing those heterogeneities generates the most value for customers. In contrast, expanding the solution space further by customizing attributes where customer needs do not fall too far apart adds little value. At some point, adding more options reduces customer value. Theory suggests an inverted u-shape of product variety and customer value [21]. Hence, it is crucial to identify where customer needs diverge and where customer needs are similar. Thus, a clearly defined solution space allows to address heterogeneous customer needs and improve efficiency simultaneously [13]. Understanding customer needs is a crucial part of solution space development. As customer needs can change over time, firms need to analyze those constantly and adjust the solution space.

To deliver products within the developed solution space, firms need to integrate a robust process design. Robust process design is a firm's "capability to reuse or recombine existing organizational and value-chain resources" [16]. Implementing a flexible and modular process design is a common way to design robust processes [22]. The key is to rearrange processes to allow product variation while ensuring near-mass production efficiency and reliability.

The third capability that supports a firm's efforts to achieve Mass Customization is customer integration [13], [16]. This capability refers to a firm's ability to support customers in identifying the product attributes that meet their needs. Firms need to minimize the complexity of choice, as evaluation options come with costs for customers [21]. In extreme cases, the cost of choice can outweigh the additional benefit from having that choice [23]. Therefore, companies

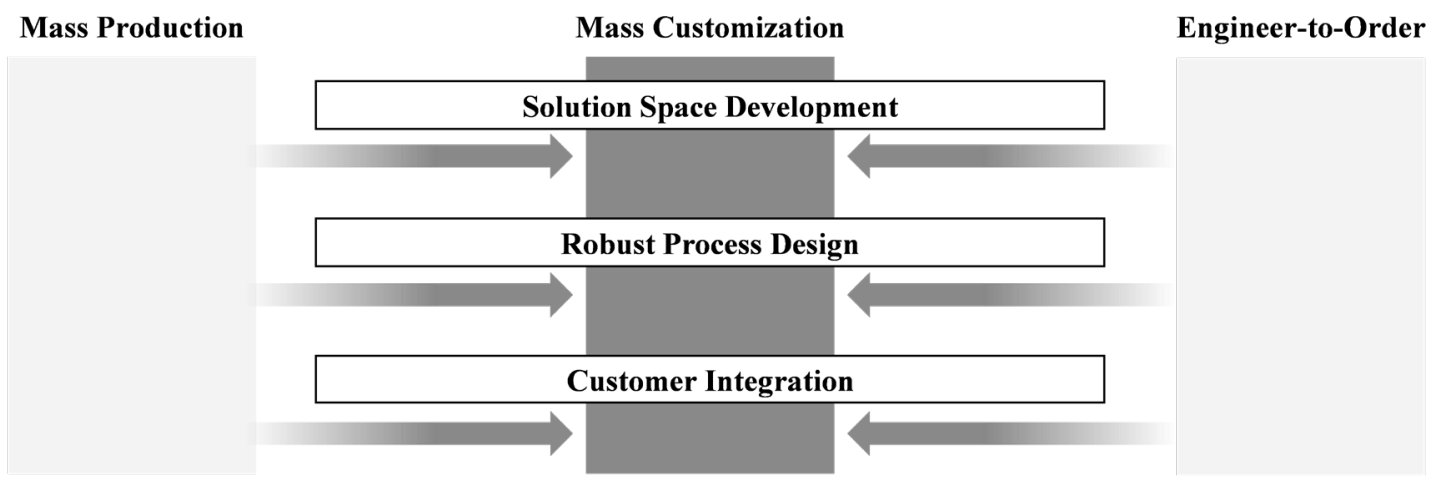

Figure 1. Continuum from mass production to engineer-to-order production 
need to carefully navigate customers' choices in a way that supports the customer toward finding their custom product.

\subsection{High-order Mass Customization Capabilities and Micro-Foundations}

Teece, Pisano, \& Shuen [24] present a firm's overall capabilities in a hierarchical structure, where high-order dynamic capabilities are underpinned by micro-foundations, which are based on operational capabilities [25]. Operational capabilities are organizational routines and managerial skills that lay the ground for business processes and keep the business running. Micro-foundations of dynamic capabilities enable firms to adapt and adjust their operational capabilities. High-order dynamic capabilities are the strategic modification of operational capabilities to address opportunities, e.g., [24]. These high-order dynamic capabilities include competencies for sensing, seizing, and transforming, which enables the firm to be innovative and responsive to the environment, e.g., [17], [26]. Firms that frequently adapt and adjust their operational capabilities develop micro-foundations through learned patterns that ultimately enable them to build high-order dynamic capabilities [25].

Similarly, Salvador et al. [16] present three MC-capabilities at the strategic level, which are rather abstract and, hence, represent high-order MC-capabilities. Firms might find it challenging to build high-order MC-capabilities as they lack concrete guidance on how to do so. Following Teece et al. [24], we structure MC-capabilities into high-order MC-capabilities and micro-foundations of MC-capabilities. Similarly, the Mass Customization literature refers to micro-foundations of MC-capabilities as MC-enablers (e.g. [12], [27], [28], [29]). Researchers have urged for the need of implementation guidelines for Mass Customization (e.g., [30]).

We aim at introducing micro-foundations of MC-capabilities, which enable firms to adapt and adjust their operational capabilities. These micro-foundations enable firms to ultimately build three high-order MC-capabilities, namely solution space development, robust process design, and customer integration.

\section{Research approach}

We examine the micro-foundations of MC-capabilities in the context of a business network in the German textile industry in an embedded case study. The investigated case is the network, while the firms represent the embedded units of analysis [31]. To present our research approach, we first describe the case and then how data was collected and analyzed.

\subsection{Case Description}

Four SMEs that are positioned at different stages of the textile value chain constitute the investigated case. Our case includes one weaving firm, one knitting firm, one textile finishing firm, and one coating firm. Thus, the level of analysis is the production network, not the four individual firms, which makes our case a single embedded case [31]. The weaving firm and the knitting firm produce textile fabrics using different technologies. The produced textile is then passed on to the finishing firm who cuts, washes, fixes, dries, and irons textiles. For particular uses, the textiles need additional coating; this is where the coating firm engages.

The firms in the network can each be a customer or supplier for each other. However, they can also act as competitors. For example, the weaver and knitter, as well as the coater and finisher can substitute each other's services. Thus, the companies in the network have a classic coopetition relationship. Upstream of the network in the value chain are raw material suppliers, like chemical or yarn manufacturers, and downstream of the network are industrial customers, e.g., from the automotive industry or the medical sector. Figure 2 shows the interaction of the four companies in the production network of our case study.

The produced textiles are not based on components or parts which can be assembled on a modular basis. Instead, the processes are reconfigured to produce a textile, which supports the notion that the firms are actors in the process industry. Due to the fragmented nature of the value chain, the firms are heavily dependent on the other firms along the value chain. The investigated firms serve business customers with technical textiles (i.e., the firms operate in a B2B context). The firms have focused on serving a niche market demanding highly complex textiles with dedicated purposes requiring custom solutions. With a deep understanding of customer needs and great experience, the firms translate the needs into customized textile solutions. The firms engage in textile sampling and research and development activities if existing textile solutions cannot meet customers' needs.

Customers value the firms' flexibility, reliability, speed, and geographical proximity. As the firms follow an engineer-to-order approach, customer requirements present a key resource, which enables the firms to develop and provide their products. The 
requirements also enable the firms to expand their repertoire of existing solutions, which can then be offered to other customers. This is a typical pattern for the engineer-to-order production approach, where the solution space is not fixed and evolves with each new customer instead. Prior to adopting an MC-approach to increase efficiency, the complex value creation process in the network was weakly standardized and barely digitized [37]. In this setting, the companies have decided to establish a digital collab-oration platform based on MC-principles [38]. The goal was to create a joint IT support to make the development of technical textiles more efficient with partners and customers.

\subsection{Data Collection and Analysis}

To identify micro-foundations of MC-capabilities, we collected data from various sources. First, we searched the field and gathered information from existing product configurators. In a second step, we conducted ten semi-structured interviews with (a) textile industry firms using product configurators and (b) developers of product configurators (cf. Table 1). We integrated the results from the field analysis into the expert interviews to validate the findings.

Identifying the micro-foundations of MC-capabilities, we followed an iterative approach. First, we analyzed the field data on product configurators. The analysis derived mainly micro-foundations of MC-ca-

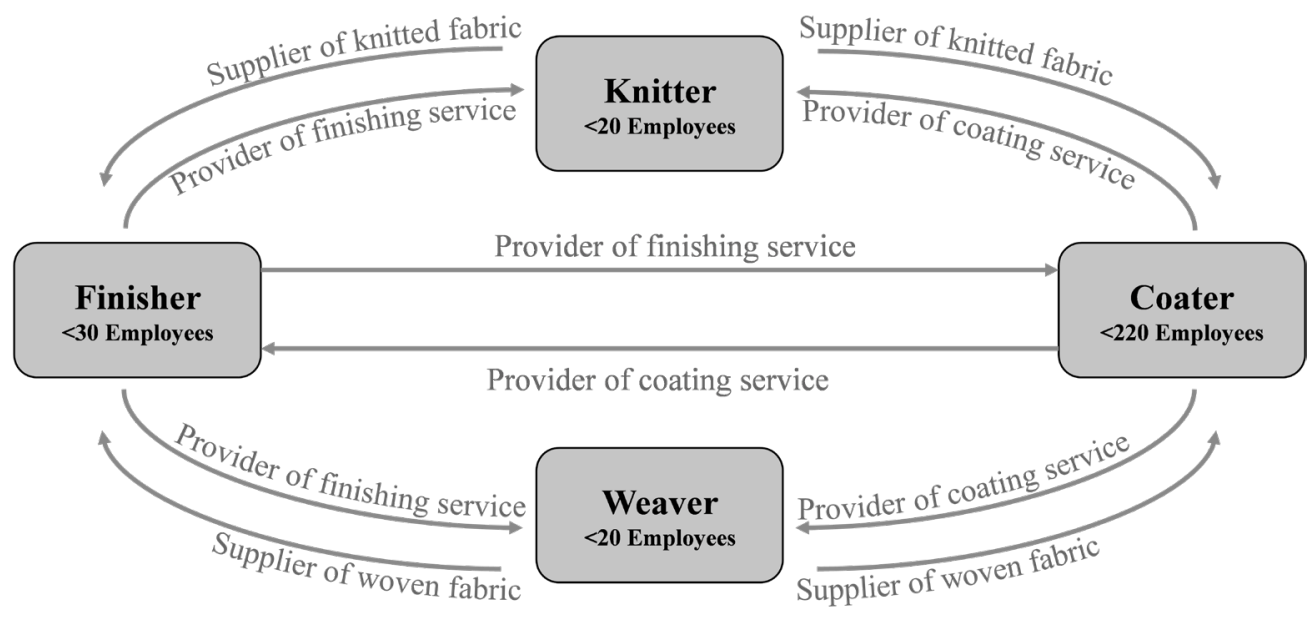

Figure 2. Single embedded case setting with four firms

Table 1. Information about the expert interviews

\begin{tabular}{|c|c|c|c|c|}
\hline Firm category & Position & Industry field & Business area & Duration \\
\hline \multirow{5}{*}{$\begin{array}{l}\text { A. Textile firm with product } \\
\text { configurator }\end{array}$} & Local IT Manager & Packaging textiles & \multirow{2}{*}{$\mathrm{B} 2 \mathrm{~B}$} & $30 \mathrm{~min}$ \\
\hline & Head of work preparation & Soft furnishings & & $46 \min$ \\
\hline & $\begin{array}{l}\text { Division Manager Variants / Pricing / } \\
\text { E-commerce }\end{array}$ & Soft furnishings & \multirow{3}{*}{$\mathrm{B} 2 \mathrm{~B}, \mathrm{~B} 2 \mathrm{C}$} & $46 \min$ \\
\hline & Managing Director & Soft furnishings & & $59 \min$ \\
\hline & Head of digital services & Soft furnishings & & $42 \min$ \\
\hline \multirow{2}{*}{$\begin{array}{l}\text { B. Software development } \\
\text { firm for product } \\
\text { configurators }\end{array}$} & Managing Director & Information Technology & \multirow{2}{*}{ B2B } & $58 \mathrm{~min}$ \\
\hline & Managing Director & Information Technology & & $50 \mathrm{~min}$ \\
\hline \multirow{3}{*}{$\begin{array}{l}\text { C. TechTex company with- } \\
\text { out product configurator }\end{array}$} & Managing Director & Technical Textiles & \multirow{3}{*}{ B2B } & $41 \mathrm{~min}$ \\
\hline & Head of Research \& Development & Technical textiles & & $47 \mathrm{~min}$ \\
\hline & Managing Director & Technical Textiles & & $29 \mathrm{~min}$ \\
\hline
\end{tabular}


pabilities for customer integration and solution space development. The workshops with MC experts added valuable insights into robust processes underlying the micro-foundations of MC-capabilities. In the first iteration, we were able to identify 46 micro-foundations of MC-capabilities. Conducting semi-structured interviews [32], we not only amained to derive additional micro-foundations from textile industry experts but also to validate the micro-foundations identified in the field study. In a second iteration, comparing interview data and field data, we dropped 26 micro-foundations as those were not suited for the B2B-context. The framework was used as an analytical lens to investigate the collaboration platform in the case of the four textile firms. Of the remaining twenty micro-foundations, nine have been implemented in the digital collaboration platform. Finally, the data from the field and literature search, the workshops, semi-structured interviews, and the case study were consolidated in a data triangulation [31] to form the final version of the framework. In addition, the investigation of the platform showed that the framework had a positive effect on the platform design, as some features, such as the property visualization of the textiles, were introduced by the four textile firms based on the insights into the micro-foundations of Mass Customization. Figure 3 shows the timeline of data collection for the case presented.

\section{Results}

The following section presents the overall structure of the developed framework for the B2B-con- text and the nine implemented micro-foundations in detail.

\subsection{The Mass Customization Micro-Foundation Framework for the Textile Industry}

In this section, we present an overview and structure of the validated twenty micro-foundations of MC-capabilities for the textile industry. We identified three overarching themes (numbered I-III) structuring four micro-foundations (numbered 1-4) of the high-order MC-capability solution space development. Developing a solution space customer heterogeneity (I) plays an important role and includes the analysis of past configurations, which enables firms to identify and account for customer heterogeneities. The solution space architecture (II) determines how the solution is built. The solution space strategy (III) determines how and under what circumstances a firm adjusts its solution space.

For the robust process design, we identified four themes (numbered IV-VII), structuring seven micro-foundations (5-11). Robust process design needs a flexible production system (IV) to enable the developed solution space. Many firms rely on modularity (V) for robust process design. The concept of robust process design includes the postponement of customization to late production stages and modular processes, which can be reconfigured. Information processing (VI) and the integration of network partners (VII) presents important themes for robust process design.

We identified four themes (numbered VIII-

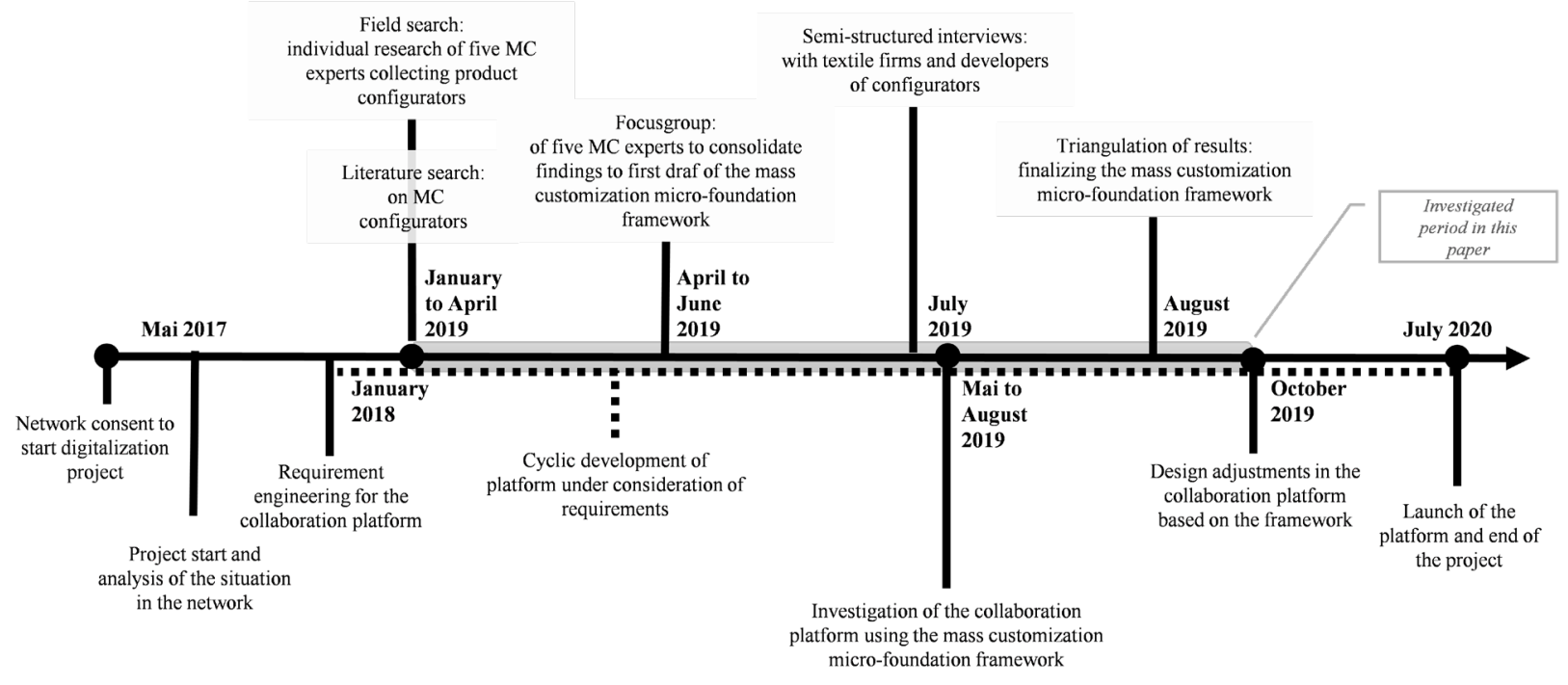

Figure 3. Timeline of the study 
$\mathrm{XI}$ ), structuring nine micro-foundations (numbered 12-20). Capturing customer need (VIII) presents a crucial step of customer integration, followed by matching the captured need (IX) to a product configuration. Furthermore, firms support customers in evaluating the product configuration (X). Firms also often support customers (XI) configuring products with help-functions and pre-configurations to find a product the fits their needs. Figure 4 illustrates the MC-micro-foundation framework.

\subsection{Micro-Foundations in Practice}

The firms in our case study identified nine micro-foundations as suited to efficiently and effectively collaborate on developing and producing custom textiles. By implementing the micro-foundations in their collaboration platform, the firms validated the importance and practical relevance of the micro-foundations in our framework, which is derived based on field and literature search and expert interviews. In this section, we first briefly describe the collaboration platform before presenting the validated micro-foundations and showing how these correspond to functions in the platform.

The collaboration platform implemented by the production network allows the firms to exchange information on production configurations, including customer requirements and the configuration of the production (who is doing what to produce the textile) along the textile value chain. The platform offers various functions for this purpose. The existing knowledge about feasible products is available in the network in a standardized digital form and serves as a knowledge database. For this purpose, valid product developments are broken down into configurations at the production process level and are stored in the platform. Furthermore, the storage of valid solutions and the corresponding process steps allow the firms to re-produce valid textile solutions quickly by reperforming the stored process steps.

The stored combination of process steps successfully carried out for the production of a textile can be used for textile development by re-configuration of existing solutions. It also serves as a basis for recommendations for the development of new textiles. The use of the collaboration platform enables the firms to take a step towards Mass Customization and standardization in the area of complex engineer-to-order products in the process industry (i.e., there is no component-based product platform and no product modules) by introducing process modularity. Hence, process modularity reduces the complexity of the production of technical textiles.

The digital collaboration platform consists of four essential components: knowledge base, process configuration component, analytics component, and overarching platform that provides the components [39]. The configuration via the platform is multilevel. First, a request for product development in the network is created using a need-based configuration. The network can then be configured (which partners are involved in the product development). Finally, the production process is configured (which production steps are carried out on which machines with which parameters by which company).

The production of textile products is complex, as it involves several firms, each performing a specific task along the value chain. Hence, the solution space of a textile depends on the limits of each of the firms involved. Configuring textiles in the early stages of the value chain allows for maximal variety. In contrast, in the late stages, the configuration is limited by the attributes set by the previous production processes. To provide customers with custom textiles that fit their needs, firms should be aware of the limits of firms upstream and downstream of the value chain.
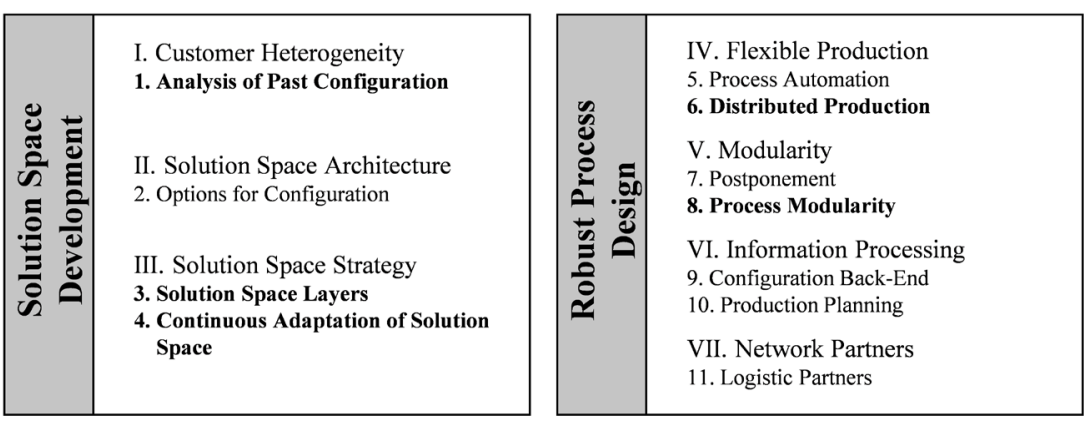

\begin{tabular}{|l|l|}
\hline & $\begin{array}{l}\text { VIII. Capture Customer Need } \\
\text { 12. Catalogue }\end{array}$ \\
13. Need-Based Configuration \\
14. Autonomous Configuration \\
15. Human Expert \\
IX. Match Need to Configuration \\
16. Sales Configuration \\
X. Evaluate Configuration \\
17. Visualization \\
18. Dynamic Pricing \\
XI. Support Customer \\
19. Help Function \\
20. Pre-configuration
\end{tabular}

Figure 4. Overview of the Mass Customization micro-foundation framework (implemented and validated micro-foundations highlighted) 
The collaboration platform integrates the solution space of the partners, enabling users to configure textiles considering the solution space along the value chain. Three micro-foundations were found suitable to define the solution space of the textile production network: solution space layers, analysis of past configurations, and continuous adaption of solution space.

Defining different solution space layers allows firms to vary the degree of customization. In our case, we identified four solution space layers in the platform. In the first layer, customers can choose from existing textile configurations (pre-configurations). Those products can be easily produced, as the production parameters (i.e., inputs, machine settings) are known and do not require textile sampling. The second layer also allows customers to choose from existing configurations but request simple changes (e.g., the thickness of the textile, color of the textile). Simple changes can be implemented with little effort. In the third layer, customers can freely configure textiles within the solution space. The firms engage in textile sampling to find a suitable solution and deliver the product. If the samples do not satisfy, the firm and the customer can decide to engage in research and development activities to perfectly meet the customer's needs, which is the fourth layer of the solution space.

The analysis of past configurations is found to be a micro-foundation of solution space development among mass customization firms. Firms analyze past configurations to understand customer needs bet- ter. Past configurations can provide favored configurations, which can be used as pre-configurations. Pre-configurations that meet many customers' needs are beneficial for customers and firms alike. Customers save time configuring a product. The firms benefit from economies of scale since the pre-configurations will be ordered more often as they meet many customers' needs. They can pass on the cost savings to the customers, which will make the product cheaper and even more attractive compared to going through a configuration process. Besides deriving pre-configurations from past configurations, the platform implements a matching algorithm. Users can configure a textile, but instead of requesting a custom product, they can request to compare their configuration with existing solutions. The algorithm lists past configurations similar to their request with information on the degree of similarity and where the differences are (cf. Figure 5). In the "Status" column, the platform shows whether the development has been successfully completed (check symbol), is still being processed (form symbol) or has been aborted (X symbol). The "Status" and "Match" columns help firms to estimate whether the feasibility of the new configuration is high (high similarity to successfully completed textile developments), or low (high similarity to failed textile developments). Similar to this micro-foundation, the automatic identification of similarities across products is a promising practice in engineer-to-order approaches [33].

Successful providers of custom products lim-

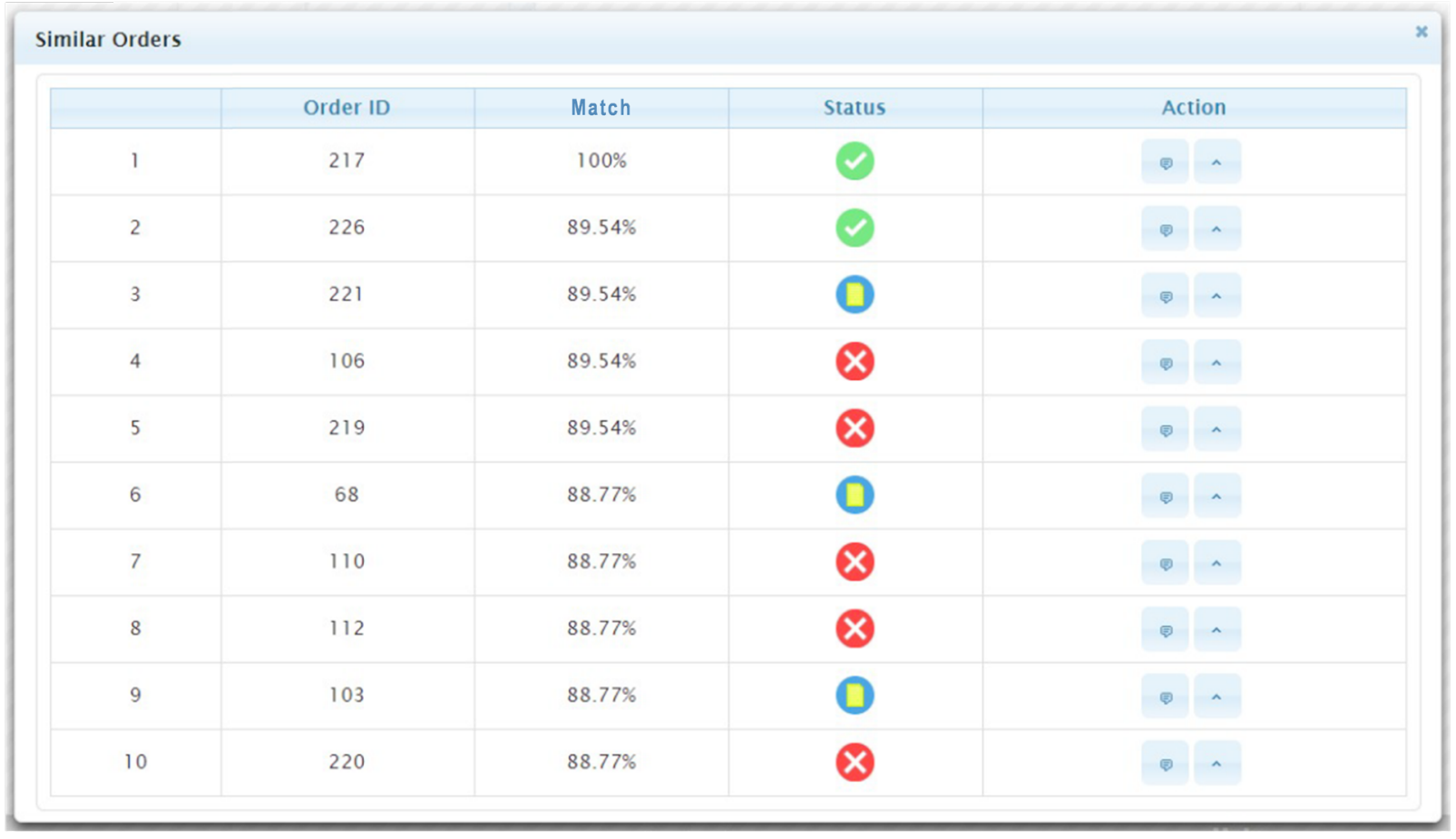

Figure 5. Analysis of past configurations on the collaboration platform 
it their solution space to reduce complexity. At the same time, they continuously adapt their solution space to changing customer needs. Those changes can be seasonal or follow long-term trends. A longterm trend in the German textile industry is the focus on technical textiles as opposed to home and apparel textiles. The firms in our case study follow this trend. They constantly evaluate whether they need to adjust their solution space to emerging needs in the textile industry. Those considerations often take place when customers' needs cannot be met using existing resources. The limitations can be due to machine specifications or due to limited knowledge. Knowledge is regularly expanded through research and development activities, which constantly enlarges the solution space and feeds the platform with pre-configured products. Solution space limits based on machine specifications can be adjusted within the platform by the firms through an administration function. The function is shown in Figure 6. The firms can add or remove configuration options (e.g., a firm can decide to produce only textiles of a single color and, hence, remove the configuration option to choose from dif-ferent colors) or adjust the range of the configura-tion options (e.g., add or remove colors, increase the maximum temperature resistance).

The investigated production network possesses capabilities to ensure a robust process design. The developed collaboration platform supports a robust process design by integrating distributed production and process modularity.

Distributed production - also known as cloud pro-

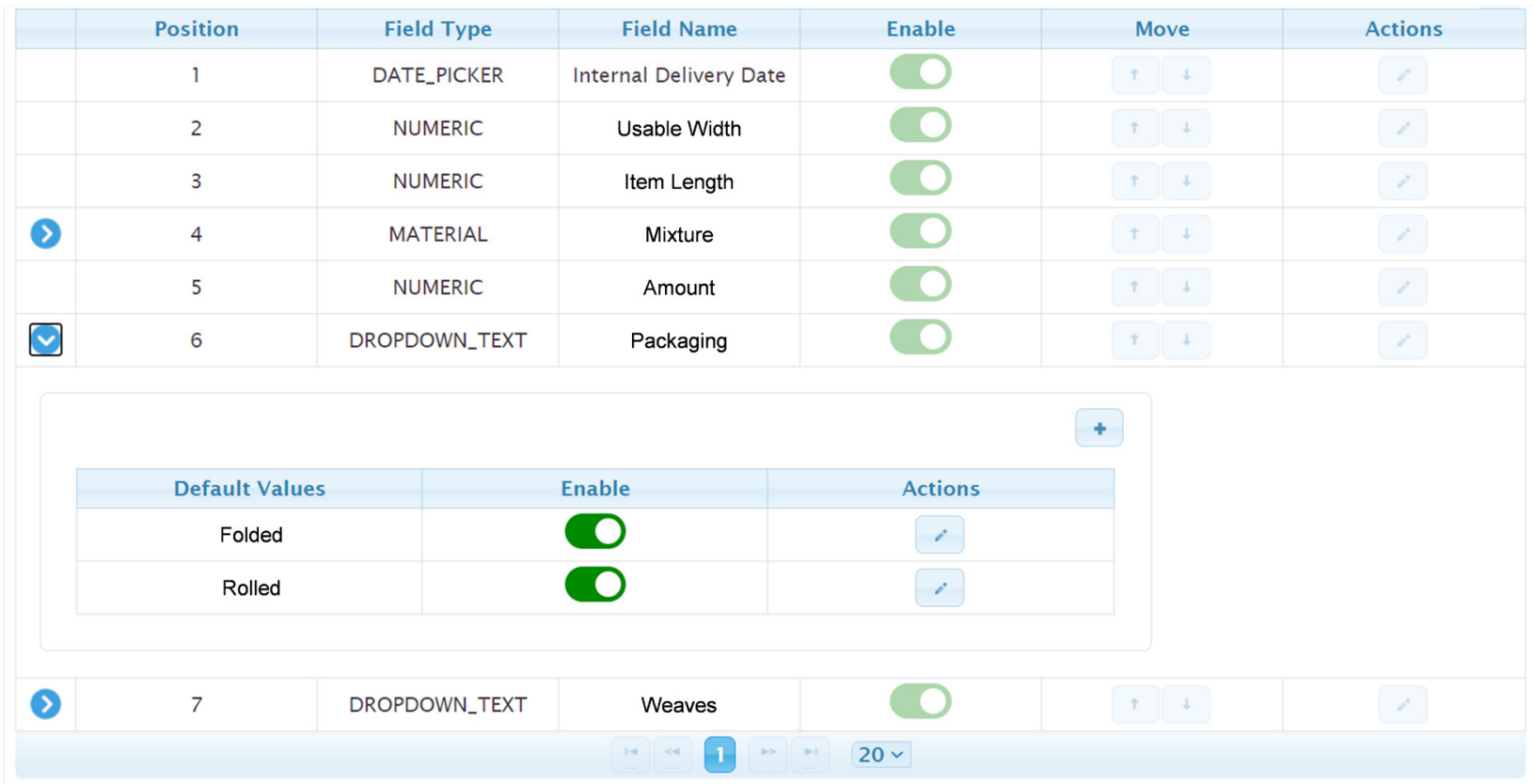

Figure 6. Continuous adaptation of solution space on the collaboration platform

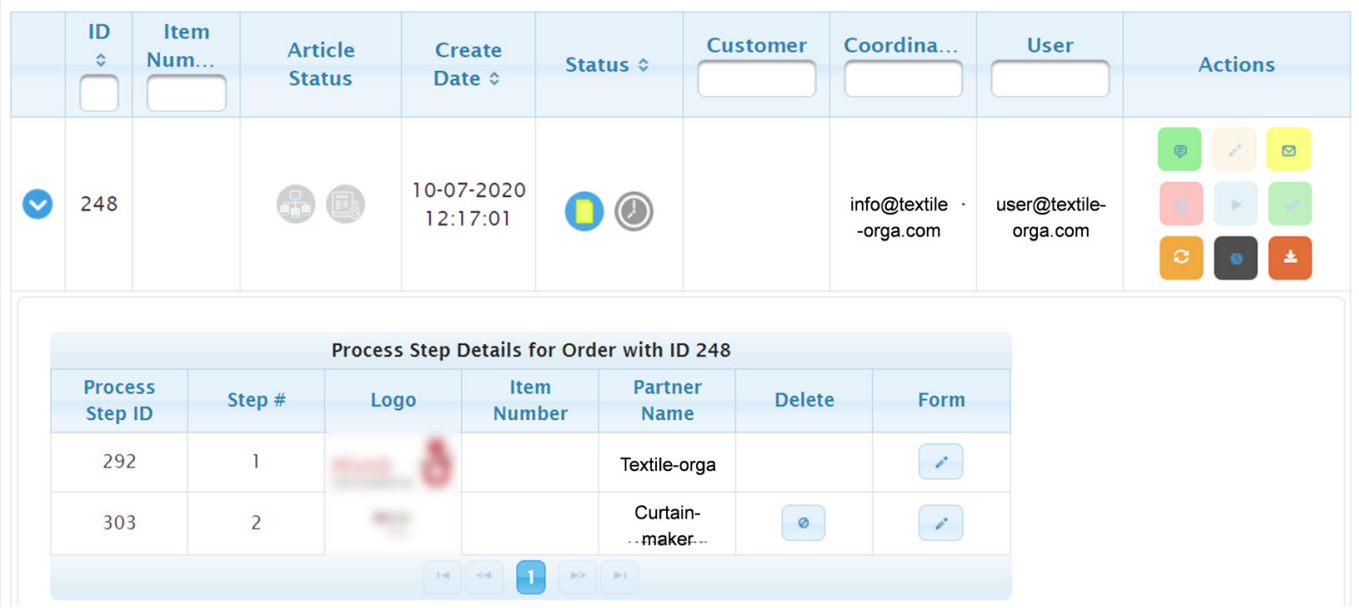

Figure 7. Distributed production on the collaboration platform 
ducing or local manufacturing - is a way to decentralize production by utilizing production resources that are geographically spread and coordinated through IT solutions. The firms in this case study rely on distributed production as each firm performs one step along the value chain. Even though each firm's production step is essential, none of the steps alone is sufficient to produce an entire textile.

The configuration platform enables the firms to coordinate their production and development of custom textiles in an integrated system. A firm receiving an order (coordinator) can add firms from the textile network needed to fulfill the order. The coordinator also suggests a production plan (e.g., who is doing what and in which order). The firms added by the coordinator automatically receive a request, which they can accept, reject, or suggest changes to (e.g., postpone the production by some days). Figure 7 shows how distributed production can be coordi-nated in the configuration platform. The upper part of the figure shows an order coordinated by firm A. The lower part shows a simplified production plan listing all firms needed to fulfill the order (i.e., firm A and firm B).

Process modularity enables firms to reconfigure their processes to meet customer needs [34] and is established in the production of custom products. For example, the finishing firm's production line is conceptualized in a way that single process steps can effortlessly be skipped. If necessary and economically reasonable, the finishing firm temporally adjusts the production line beyond simply skipping process steps (e.g., borrow a machine to add a process step for a high-volume order). Similarly, the coating firm

\begin{tabular}{|l|l|}
\hline Trim \\
\hline Textile Bleach \\
\hline Textile Dye
\end{tabular}

Figure 8. Process modularity on the collaboration platform

\section{Configuration of New Textile Developments

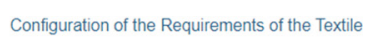

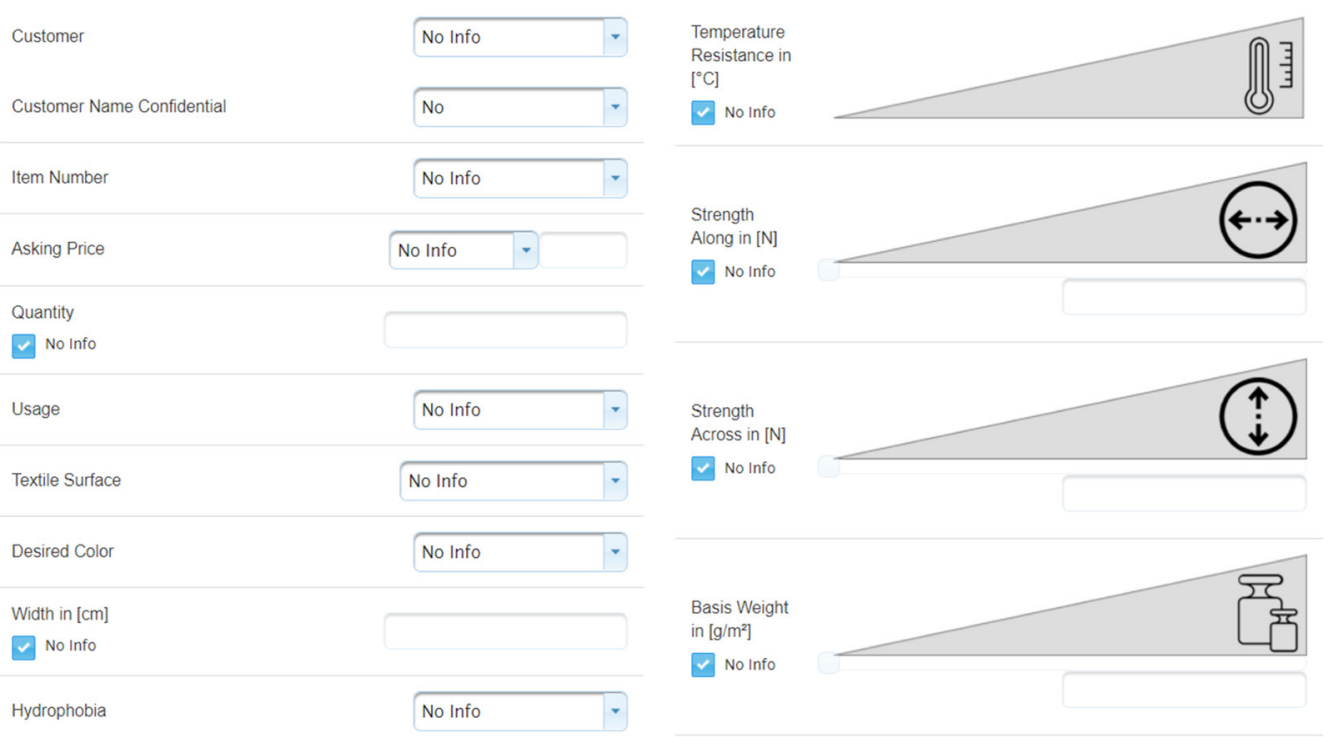

Figure 9. Need-based configuration on the collaboration platform 
can flexibly configure single production processes.

To enable creating a detailed production plan, the textile network digitalized their modular processes (i.e., the firms defined their modular processes on the platform). Figure 6 shows the different process modules of the finishing firm. The coordinator of an order can select single process steps creating a detailed production plan. The finishing firm can adjust the production plan when receiving a production request.

Coordinating the modular processes on the configuration platform allows distributed production while taking the machine capacities and processes of each partner needed to fulfill an incoming order into account. Hence, enabling more efficient and reliable coordination of production resources.

Customer requirements constitute a key resource for the investigated firms. To capture the requirements, firms need to integrate the customer. Customer integration is enabled by the configuration front-end of the collaboration platform. The platform implements four micro-foundation of customer integration: need-based configuration, sales configuration, pre-configuration, and visualization.

A need-based configuration is an approach where customers are asked to state their needs, preferences, and expected outcomes, i.e., the textile's intended use, the attributes the textile must have (e.g., waterproof, scratch-resistant). The need-based configuration as opposed to a parameter-based configuration, where customers are asked to state specific input parameters. The configurator implemented by the textile production network does not ask the customer to choose a yarn, a finishing chemical, and a coating but rather asks what requirements the textile needs to fulfill (cf. Figure 9).

In B2C-contexts, one often find web configurators for fast-moving consumer goods (e.g., food, cosmetics). Those configurators are operated by the customer to create their desired product and order it directly via the website. For highly complex products, where specific know-how is necessary to operate the configurator, sales configuration is a common approach. In a sales configuration, a sales employee is operating the configurator to capture the customers' requirements. Configuring textiles is highly complex. Therefore, the primary use of the configurator is the sales configuration. However, firms can give access to the configurator to knowledgeable customers.

Pre-configuration is a thriving practice for integrating customers. Instead of starting from scratch, customers are offered pre-configured products. In the investigated case, the firms add configured textiles continuously to the collaboration platform contributing to a growing base of pre-configurations. Users of the configurator (customers or sales employees) can access the order history to re-order pre-configured products.

Re-ordering pre-configurations refers to the first solution space layer. The platform allows the user

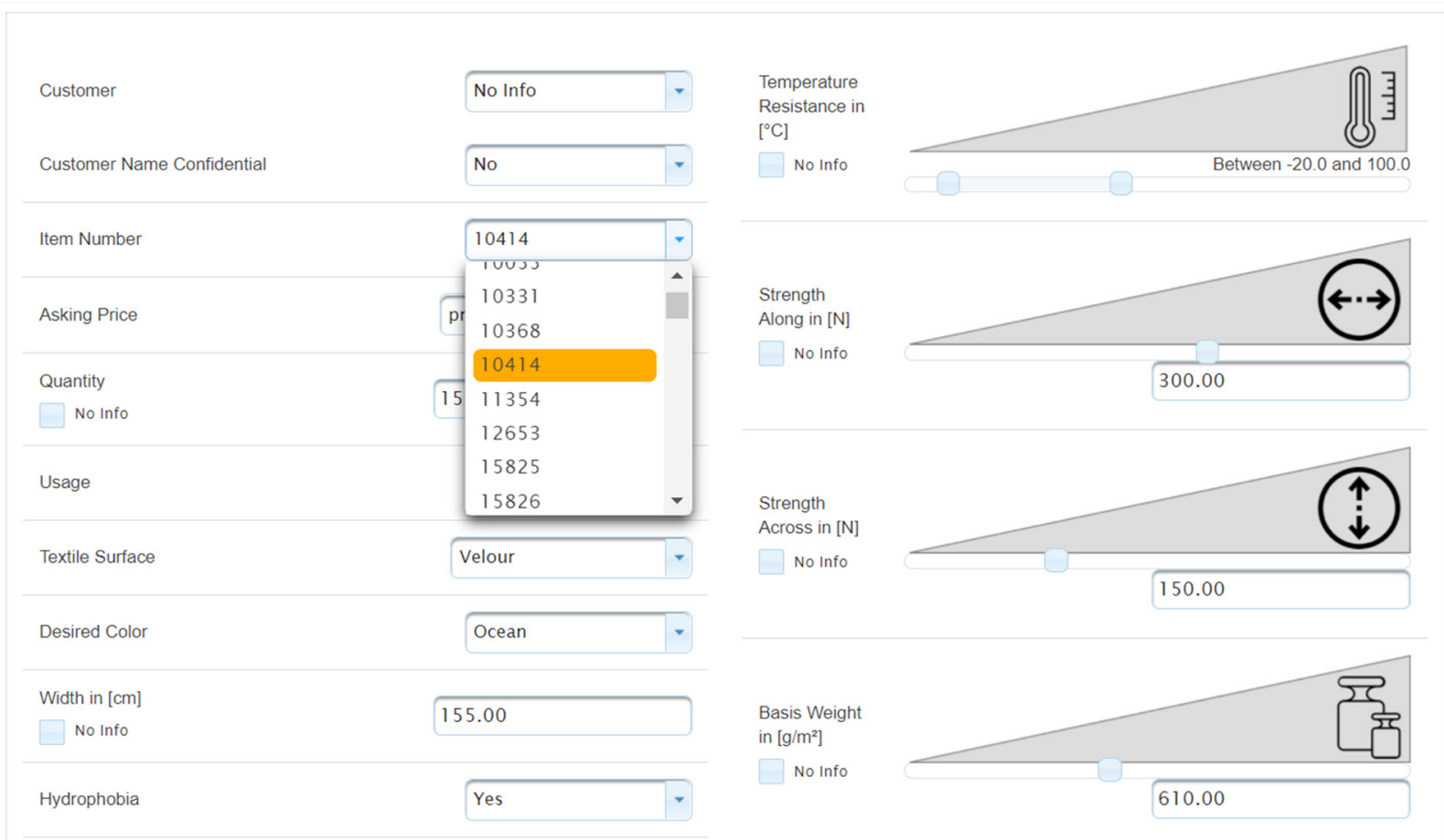

Figure 10. Pre-configuration on the collaboration platform 
to make adjustments to the pre-configured products to meet specific customer needs. If customers make simple changes, they enter the second solution space layer. By making changes that require textile sampling (i.e., firms making a couple of iterations following a trial-and-error approach to meet customer needs), customers enter the third solution space layer. Figure 10 shows the order of a pre-configuration. The user chooses from a number of pre-configurations in the field "item number" (first solution space layer). By selecting the item number, the configurator automat-ically sets all options. Users can then check whether the specifications satisfy their needs. If not, they can make adjustments and thereby enter the second or third solution space laydusers can also choose not to rely on pre-configurations but rather start the configuration from scratch and insert their requirements (compare Figure 9, need-based configuration). From here, users can either enter the fourth solution space layer and engage in research and development activities with the firms, or they can use the function "analysis of past configurations." Suppose the analysis finds a configuration that satisfies users' needs. In that case, users can choose to order the pre-configuration (first solution space layer) or again make adjustments to the configuration (second or third solution space layer). The combination of pre-configuration, solution space layers, and analysis of past configurations allows saving research and development costs whenever possible. A growing database of pre-configurations will increase the chances that users order pre-configured products.

The visualization of configured products is an es- tablished practice for consumer goods. Visualizing configured textiles, however, is rather difficult and often does not add essential value. Finishing a textile, for example, does not change its visual appearance. However, it is possible to visualize some key features of the textile to show these important attributes at a glance and for easy comparison. Figure 11 illustrates how the visualization of the textile configuration is now realized in the collaboration platform. The cake chart indicates to what extent each listed attribute is pronounced; the bigger the cake piece, the more the attribute is pronounced. The cake chart in Figure 11 presents a medium weight (green piece) textile with a low to medium strength crosswise (dark blue piece), a high strength lengthwise (orange piece), a medium temperature resistance for high temperatures and low temperature resistance for low temperatures (light blue piece).

\section{Discussion}

The Mass Customization literature provides high-order MC-capabilities, which are necessary for a firm's success in pursuing an MC-strategy [16]. However, those capabilities are rather abstract and, especially for SMEs with limited time and resources, difficult to grasp [35]. Following the capability hierarchy of Teece et al. [24], we derived micro-foundations that provide actionable guidance for textile SMEs on how to build high-order MC-capabilities (i.e., solution space development, robust process design, and customer integration). Our study contributes to the Mass Customization literature by introducing an integrated framework of micro-foundation of high-order

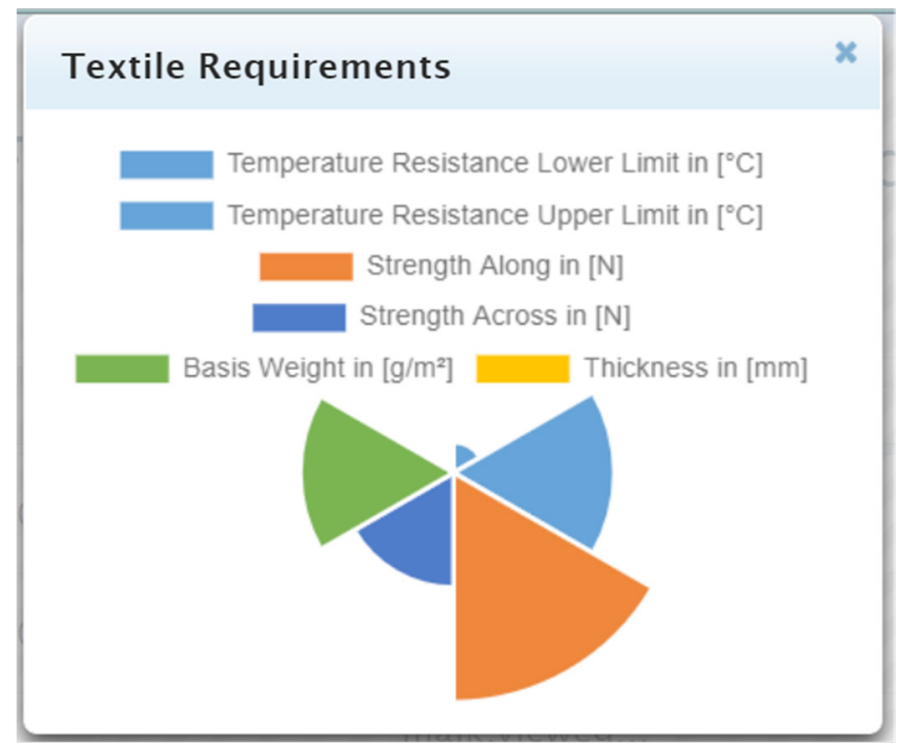

Figure 11. Visualization of the textile configuration on the collaboration platform 
MC-capabilities. Our research fills a gap between the high-order MC-capabilities of firms [16] and operational capabilities that managers in Mass Customization firms need to have [36]. An example of this interconnection of the hierarchical MC-capabilities may be as follows: At a high order, firms need the capability of robust process design. At the micro-foundation level, they may rely on distributed production, and their employees, therefore, need negotiation capabilities at an operational level [36] to coordinate distributed production between companies efficiently.

In our case study, the firms put nine micro-foundations into practice by integrating them into the digital collaboration platform. Hence, our study provides a set of nine validated micro-foundations of MC-capabilities. We contribute to the literature by highlighting the path of SMEs in fragmented process industries with an engineer-to-order approach moving towards an MC-approach (cf. Figure 1).

At the same time, the validated micro-foundations can be applied in practice, as they can serve textile networks striving for MC-efficiency to develop high-order MC-capabilities. Furthermore, the nine validated micro-foundations can also be transferred to other process industries (e.g., chemical and metal-processing industry) following an engineer-to-order approach. The overall framework can be used to identify additional suitable micro-foundations for other B2B-contexts. The actionable micro-foundations can help firms to develop high-order MC-capabilities.

Our study is not free of limitations. We present micro-foundations of MC-capabilities based on a single case study. The transferability of the micro-foundations to other industries is therefore not necessarily given. By carefully mapping out the characteristics of our case (i.e., engineer-to-order approach, SMEs, fragmented network production, process industry), we defined the borders of the transferability of our results. However, we aim - and also encourage other researchers - to empirically validate the overall micro-foundation framework.

For future research, we aim at validating the effect of the presented, implemented micro-foundations. Did the efficiency and effectiveness of the engineer-to-order approach actually increase through the use of the collaboration platform? This is a research question we aim to address in future research. Therefore, we conducted initial interviews with each firm and conceptualized a quantitative survey measuring the use of the digital collaboration platform.

\section{Conclusion}

In our case study, we present a framework structuring the micro-foundations of MC-capabilities. More specifically, we presented nine validated micro-foundations in detail, which provide fragmented process industries with an engineer-to-order approach with actionable insights on how to leverage MC-principles and develop high-order MC-capabilities. The firms of our case study developed a collaboration platform for textile product configuration entailing the nine presented micro-foundations. The collaboration platform and the implemented micro-foundations helped the firms to break down their complex processes and define their solution space. Furthermore, customer integration is standardized using the developed platform, and customer requirements can be shared along the textile value chain. Overall, the aim of the developed platform is to allow the firms to exchange information on product development and production more efficiently and more effectively in the production network. By developing MC-capabilities through the implementation of micro-foundations, the firms were able to leverage MC-principles aiming to introduce efficiency into an engineer-to-order approach for custom textiles. The digital collaboration platform has the potential to reduces development and production times, a crucial competitive advantage in the textile industry [11]. Hence, the micro-foundations of MC-capabilities embedded in the digital collaboration platform aim to strengthen the firms' competitive advantage and ensure their survival.

\section{Funding}

The research described in this paper was supported by a grant from the German Ministry for Research and Education (BMBF), project name: PROFUND (Processoriented configuration of value creation in textile networks for mass customization in SME), FKZ: $037 Z 0618$

\section{References}

[1] Federal Statistical Office Germany, "Structure of the Classification of Economic Activities, Edition 2008," Wiesbaden, 2008.

[2] C. Byrne, "Technical textiles market - an overview," in Handbook of Technical Textiles, A. R. Horrocks and S. C. Anand, Eds. Cambridge: Woodhead Publishing, 2000, pp. 1-23.

[3] I. M. Taplin and J. Winterton, "The European clothing industry: Meeting the competitive challenge," J. Fash. Mark. Manag. An Int. J., vol. 8, no. 3, pp. 256-261, 2004.

[4] R. M. Jones and S. G. Hayes, "The UK clothing industry: Extinction or evolution?, J. Fash. Mark. Manag. An Int. J., vol. 8, no. 3, pp. 262-278, 2004. 
[5] J. R. Bryson and M. Ronayne, "Manufacturing carpets and technical textiles: Routines, resources, capabilities, adaptation, innovation and the evolution of the British textile industry," Cambridge J. Reg. Econ. Soc., vol. 7, no. 3, pp. 471-488, 2014.

[6] S. Noroozi and J. Wikner, "Sales and operations planning in the process industry: A literature review," Int. J. Prod. Econ., vol. 188, no. 3, pp. 139-155, 2017.

[7] European Commission, "Country Reports on Technical Textiles ('TT') in Brazil, Japan, South Korea and USA," 2016.

[8] WTO Report, "World Trade Statistical Review," Geneva, Switzerland, 2019.

[9] Y. Kristianto, P. Helo, and R. J. Jiao, "A system level product configurator for engineer-to-order supply chains," Comput. Ind., vol. 72, no. C, pp. 82-91, 2015.

[10] C. Hicks, T. McGovern, and C. F. Earl, "Supply chain management: A strategic issue in engineer to order manufacturing," Int. J. Prod. Econ., vol. 65, no. 2, pp. 179-190, 2000.

[11] N. J. Hodges and A. N. Link, Knowledge-Intensive Entrepreneurship: An Analysis of the European Textile and Apparel Industries. Cham: Springer, 2018.

[12] F. S. Fogliatto, G. J. C. Da Silveira, and D. Borenstein, "The mass customization decade: An updated review of the literature,” Int. J. Prod. Econ., vol. 138, no. 1, pp. 14-25, 2012.

[13] F. Piller, K. Moeslein, and C. Stotko, "Does mass customization pay? An economic approach to evaluate customer integration," Prod. Plan. Control, vol. 15, no. 4, pp. $435-444,2004$.

[14] L. L. Zhang, "Product configuration: a review of the state-ofthe-art and future research," Int. J. Prod. Res., vol. 52, no. 21, pp. 6381-6398, 2014.

[15] E. von Hippel, "User Toolkits for Innovation," J. Prod. Innov. Manag., vol. 18, no. 4, pp. 247-257, 2001.

[16] F. Salvador, P. M. de Holan, and F. Piller, "Cracking the Code of Mass Customization," MIT Sloan Manag. Rev., vol. 50, no. 3, pp. 71-78, 2009 .

[17] D. J. Teece, "The foundations of enterprise performance: Dynamic and ordinary capabilities in an (economic) theory of firms," Acad. Manag. Perspect., vol. 28, no. 4, pp. 328352, 2014.

[18] F. Salvador, P. de Holan, and F. Piller, "Cracking the Code of Mass Customization,” MIT Sloan Manag. Rev., vol. 50, no. 3, pp. 71-78, 2009.

[19] K. M. Eisenhardt, N. R. Furr, and C. B. Bingham, "Micro foundations of performance: Balancing efficiency and flexibility in dynamic environments," Organ. Sci., vol. 21, no. 6, pp. 1263-1273, 2010.

[20] M. M. Tseng and J. Jiao, "Mass customization," Handb. Ind. Eng., vol. 3, pp. 684-709, 2001.

[21] C. Huffman and B. E. Kahn, "Variety for sale: Mass customization or mass confusion?,” J. Retail., vol. 74, no. 4, pp. 491-513, 1998.

[22] B. J. Pine, B. Victor, and A. Boynton, "Making mass customization work," Harv. Bus. Rev., vol. 71, no. 5, pp. 108-111, 1993.

[23] R. Desmeules, "The impact of variety on consumer happiness: Marketing and the tyranny of freedom," Acad. Mark. Sci. Rev., vol. 12, no. 1, pp. 1-18, 2002.

[24] D. J. Teece, G. Pisano, and A. Shuen, "Dynamic capabilities and strategic management,” Strateg. Manag. J., vol. 18, no. 7, pp. 509-533, 1997.

[25] C. Helfat and S. Winter, "Untangling Dynamic and Operational Capabilities: Strategy for the (N)Ever-Changing World,” Strateg. Manag. J., vol. 32, pp. 1243-1250, 2011.

[26] D. J. Teece, "Business models and dynamic capabilities," Long Range Plann., vol. 51, no. 1, pp. 40-49, 2018.
[27] R. Silvestro and P. Lustrato, "Exploring the 'mid office' concept as an enabler of mass customization in services," Int. J. Oper. Prod. Manag., vol. 35, no. 6, pp. 866-894, 2015.

[28] N. Levesque and H. Boeck, "Proximity Marketing as an Enabler of Mass Customization and Personalization in a Customer Service Experience," in Managing Complexit, J. Bellemare, S. Carrier, K. Nielsen, and F. T. Piller, Eds. Cham: Springer, 2017, pp. 405-420.

[29] G. J. C. Da Silveira, D. Borenstein, and F. S. Fogliatto, "Mass customization: Literature review and research directions,” Int. J. Prod. Econ., vol. 72, no. 49, pp. 1-13, 2001.

[30] N. Suzić, E. Sandrin, S. Suzić, C. Forza, A. Trentin, and Z. Anišić, "Implementation guidelines for mass customization: A researcher-oriented view," Int. J. Ind. Eng. Manag., vol. 9, no. 4, pp. 229-243, 2018.

[31] R. K. Yin, Case Study Research and Applications: Design and Methods. Los Angeles: SAGE Publications, Inc., 2018.

[32] M. D. Myers and M. Newman, "The qualitative interview in IS research: Examining the craft," Inf. Organ., vol. 17, no. 1, pp. 2-26, 2007.

[33] S. Shafiee, K. Kristjansdottir, and L. Hvam, "Automatic Identification of Similarities Across Products to Improve the Configuration Process in ETO Companies,” Int. J. Ind. Eng. Manag., vol. 8, no. 3, pp. 167-176, 2017.

[34] Q. Tu, M. A. Vonderembse, T. S. Ragu-Nathan, and B. Ragu-Nathan, "Measuring modularity-based manufacturing practices and their impact on mass customization capability: A customer-driven perspective,” Decis. Sci., vol. 35, no. 2, pp. 147-168, 2004.

[35] I. Mendikoa, M. Sorli, J. I. Barbero, A. Carrillo, and A. Gorostiza, "Collaborative product design and manufacturing with inventive approaches," Int. J. Prod. Res., vol. 46, no. 9, pp. 2333-2344, 2008.

[36] A. Trentin, T. Somià, E. Sandrin, and C. Forza, "Operations managers' individual competencies for mass customization," Int. J. Oper. Prod. Manag., vol. 39, no. 9-10, pp. 10251052,2019

[37] L. K. Grafmüller, S. Hankammer, S. Hönigsberg, and H. Wache, "Developing complex, mass-customized products in SME networks: Perspectives from co-creation, solution space development, and information system design," Int. J. Ind. Eng. Manag., vol. 9, no. 4, pp. 215-227, 2018.

[38] S. Hönigsberg, B. Dinter, and H. Wache, "The Impact of Digital Technology on Network Value Co-creation," in 53rd Hawaii International Conference on System Sciences, 2020, pp. 5233-5242.

[39] S. Hönigsberg, "A Platform for Value Co-Creation in SME Networks," in Designing for Digital Transformation. Co-Creating Services with Citizens and Industry. DESRIST 2020. Lecture Notes in Computer Science, vol 12388, S. Hofmann, O. Müller, and M. Rossi, Eds. Cham: Springer, 2020, pp. 285-296. 